\title{
MANEJO DE LA MIGRACIÓN POSTERIOR DE LOS DISPOSITIVOS INTERSOMÁTICOS COLOCADOS POR VÍA POSTERIOR (PLIF). ANÁLISIS BIBLIOGRÁFICO
}

\author{
CONDUTA NA MIGRAÇÃO POSTERIOR DOS DISPOSITIVOS INTERSOMÁTICOS COLOCADOS \\ POR VIA POSTERIOR (PLIF). ANÁLISE DA LITERATURA
}

\author{
HANDLING OF THE LATER MIGRATION OF THE INTERSOMATIC DEVICES PLACED BY LATER \\ ROUTE (PLIF). BIBLIOGRAPHICAL ANALYSIS
}

Pedro luis Bazán ${ }^{1}$, Alvaro Enrique Borri ${ }^{1}$, Enrique Gonzalo Bovier ${ }^{1}$, Benedetti Cosentino ${ }^{1}$, Sebastián Juan ${ }^{1}$

\begin{abstract}
RESUMEN
Objetivo: Se tiene la finalidad de presentar el cuadro clínico de tres casos de esta complicación y analizar metodológicamente los trabajos existentes en la bibliografía. Método: Tres pacientes operados de columna lumbar a quienes se les realizó una artrodesis circunferencial y que presentaron en el posoperatorio, después de bastante tiempo, nueva sintomatología relacionada con la migración de uno o de los dos PLIF. Resultados: Se realizó mediante el abordaje por vía anterior retroperitoneal, para disectomía, retiro de material migrado y colocación de un dispositivo intersomático anterior. En el primer caso se asoció un abordaje posterior para el retiro del material de osteosíntesis. Se realizó una búsqueda bibliográfica para analizar los datos sobre el retiro según parámetros de evidencia científica. Conclusiones: No existen publicaciones de Evidencia tipo 1 o 2 que marquen directivas claras. La presencia de fibrosis posquirúrgica orientaría al abordaje por vía anterior en el retiro de los dispositivos intersomáticos y así evitaría las lesiones del saco dural y la elongación radicular. La dificultad del manejo vascular y el riesgo de eyaculación retrógrada en pacientes del sexo masculino son parámetros a tener en cuenta para optar por el abordaje posterior. El retiro de los PLIF migrados a posterior, que causen sintomatología dolorosa y/o deficitaria, se convierte en una práctica altamente exigente y de manejo dificultoso.
\end{abstract}

Descriptores: Vértebras lumbares; Fusión vertebral; Procedimento quirúrgico minimamente invasivo.

RESUMO

Objetivo: Apresentar o quadro clínico de três casos dessa complicação e analisar metodologicamente os trabalhos presentes na literatura. Método: Três pacientes submetidos à cirurgia da coluna lombar na qual se realizou artrodese circunferencial e que apresentaram nova sintomatologia no pós-operatório tardio, relacionada com a migração de um ou dos dois PLIF. Resultados: Realizou-se por acesso retroperitoneal anterior, para discectomia, a retirada do material que migrou e a colocação de um dispositivo intersomático anterior. No primeiro caso, associou-se acesso posterior para a retirada do material de osteossíntese. Efetuou-se uma busca na literatura para analisar os dados sobre a retirada de acordo com parâmetros de evidência científica. Conclusões: Não existem publicações de evidências do tipo 1 ou 2 que marquem diretrizes claras. A presença de fibrose pós-cirúrgica orientaria o acesso anterior na retirada dos dispositivos intersomáticos e, assim, evitaria as lesões do saco dural e o alongamento radicular. A dificuldade da manipulação vascular e o risco de ejaculação retrógrada em pacientes do sexo masculino são parâmetros a considerar ao escolher o acesso posterior. A retirada dos PLIF migrados para posterior que causam sintomatologia dolorosa e/ou deficiência torna essa prática altamente exigente e difícil.

Descritores: Vértebras lombares; Fusão vertebral; Procedimento cirúrgico minimamente invasivo.

\begin{abstract}
Objective: With the objective to present the clinical picture of three cases with this complication and analyze methodologically the works present in the bibliography. Method: Three operated patients of lumbar column that received a circumferential arthrodesis and presented in postoperative remote a new symptomatology related to the migration of one or of both PLIF. Results: It was made by means of the boarding by retroperitoneal previous route, for discectomy, retirement of migrated material and positioning of a previous intersomatic device. In the first case It was associated a later boarding for the retirement of the osteosynthesis material. A bibliographical search was made to analyze the data on the retirement according to parameters of scientific evidence. Conclusions: Publications of Evidence type 1 or 2 do not exist that mark clear orders. The presence of post-surgical fibrosis would orient to the boarding by previous route in the retirement of the intersomatic devices and thus to avoid the injuries of the dural coat and the radicular stretching. The difficulty of the vascular handling and the risk of retrograde ejaculation in patients of male sex are parameters to consider when deciding on the later boarding. The retirement of the PLIF migrated to later that cause painful symptomatology and/or deficit in a practice becomes highly demanding and difficult to handle.
\end{abstract}

keywords: Lumbar vertebrae; Spinal fusion; Surgical procedures minimally invasive. 


\section{INTRODUCCIÓN}

El uso de dispositivos intersomáticos por vía posterior (PLIF) comenzó a mediados de 1940 con Cloward apud Chen et al. ${ }^{1}$, fue dejado de lado por las severas complicaciones de la utilización de hueso, hasta que se comenzaron a utilizar las cajas, su utilización ha aumentado con el correr de los años luego de mejorar la calidad de los materiales con los que son construidos ${ }^{2}$.

Las razones de su uso pueden explicarse por causas mecánicas como son el soporte y estabilización de la columna anterior, el mayor contacto de las superficies de fusión, la restauración de la altura del espacio discal y del espacio foraminal.

La ventaja biológica sobre la fusión posterolateral es que por la compresión axial cerca del centro de rotación, favorece la artrodesis. El injerto óseo, una vez establecido, reconstituye la columna anterior adquiriendo mayor resistencia ${ }^{3}$.

Dentro de las complicaciones de su utilización se detallan las lesiones del saco dural, neuropraxia por separación radicular, subsidencia, migración posterior, pseudoartrosis e infecciones ${ }^{1}$.

La presencia de una migración posterior del dispositivo, que ocurre en el $8 \%$ de los casos y requiere revisión en el $57 \%{ }^{1}$, puede ocasionar dolor lumbar mecánico, dolor radicular por compresión y nuevos síntomas deficitarios.

Su manejo no es sencillo si ocurre luego de unos meses de su colocación, donde se puede poner en consideración su retiro por la misma vía de colocación o por vía anterior, siendo estas terapéuticas demandantes.

Una evaluación de 1680 pacientes con 3053 niveles fusionados con seguimiento de 5 años, han evaluado las causas de revisión que fueron pseudoartrosis, descompensación del segmento adyacente, re descompresión, falla del implante, espondilitis iatrogénica ${ }^{4-6}$.

En los últimos años han salido publicaciones sobre el uso de una sola caja colocada por vía posterior pero en forma diagonal, como lo muestra Zhao et al. ${ }^{7}$ donde obtuvieron buena tasa de fusión.

El objetivo de la siguiente presentación, es por un lado presentar el cuadro clínico de cuatro pacientes asistidos en el último año, que presentaron una migración posterior de uno o dos PLIF, evaluar su tratamiento quirúrgico y analizar metodológicamente los trabajos presentes en la bibliografía.

\section{MATERIAL Y MÉTODO}

Cuatro pacientes operados de columna lumbar a quienes se les realizaron una artrodesis circunferencial y que presentaron en el post-operatorio alejado nueva sintomatología relacionada con la migración de uno o de los dos PLIF, que consultaron en el último año a los autores:

El primer paciente de sexo femenino de 44 años de edad que concurre por lumbociatálgia derecha de 2 años de evolución, luego de una intervención quirúrgica por una lumbalgia de 5 años. Se le realizo una artrodesis circunferencial con tornillos y PLIF del espacio L5-S1. A su consulta se constata la migración posterior de los 2 PLIF hacia el canal comprometiendo principalmente el receso derecho en (radiografías, tomografía y resonancia), se agrega además la presencia aflojamiento de los tornillos sacros (Figura 1)

Segundo paciente del sexo masculino de 31 años de edad, con antecedente de 2 cirugías previas, la primera de ellas en Octubre de 2004 para liberación de una ciática derecha de topografía S1 que remitió por un lapso de dos meses. En Junio de 2005, se realizó nueva cirugía para artrodesis L4-S1 con tornillos pediculares y PLIF L4-L5 por deshidratación de los dos últimos espacios y hernia de disco extraforaminal L4-L5.

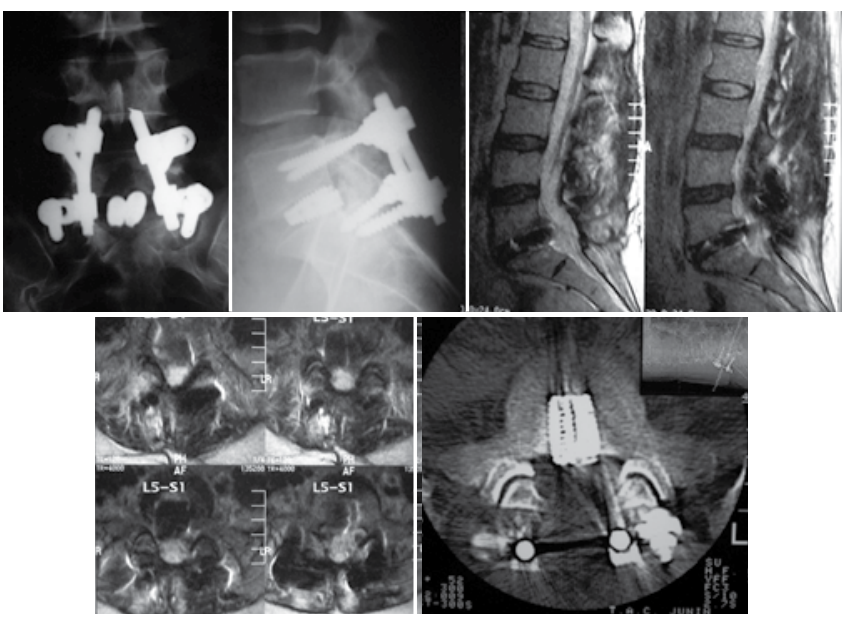

Figura 1. Estudios del paciente $\mathrm{N}^{\circ} 1$ con aflojamiento del material de osteosíntesis y migración posterior de ambas cajas.

En la actualidad lumbociatálgia derecha de topografía L5. En exámenes complementarios se constata la migración de las dos cajas al canal raquídeo (Figura 2).

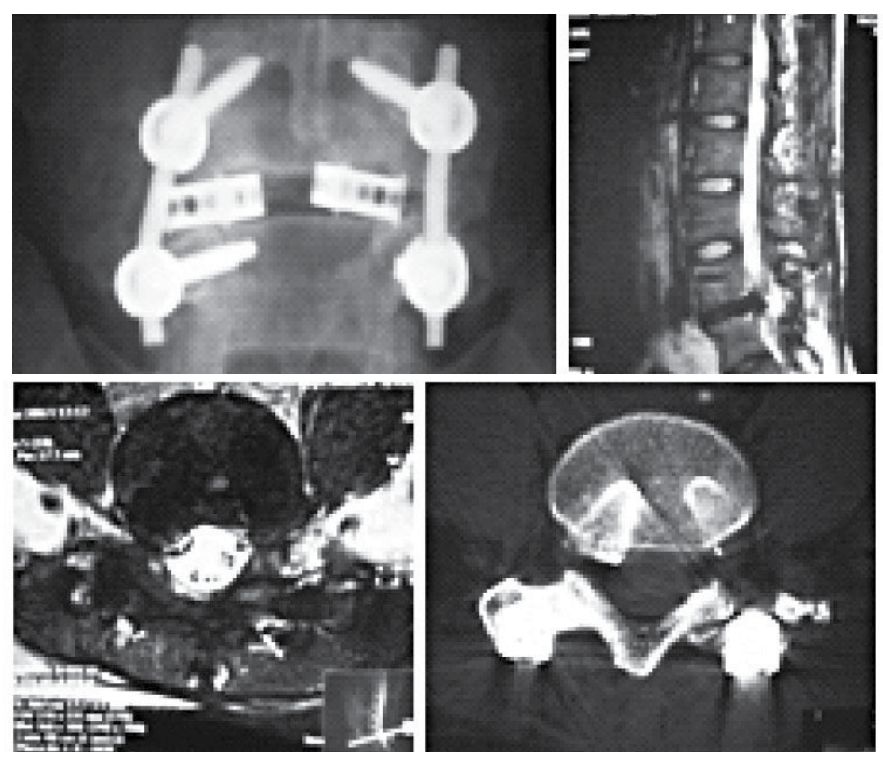

Figura 2. Imágenes del Paciente №2, que muestra la migración posterior y lateral de las cajas.

El tercer caso es un paciente del sexo masculino de 32 años de edad, que consulta por lumbociatálgia derecha recidivada, refiriendo el antecedente de una cirugía de columna lumbosacra por lumbalgia, realizada por vía posterior hacía más de un año, momento en que se realizó una liberación y fijación circunferencial mediante instrumentación con tornillos pediculares $L 4$ y $L 5$ y 2 cages para artrodesis intersomática por vía posterior (PLIF). Refiere dolor irradiado a miembro inferior inmediatamente después de la cirugía.

El cuarto caso corresponde a un paciente del sexo femenino de 46 años de edad, que consulta por lumbalgia y paresia de la $5^{\text {ta }}$ raíz lumbar derecha, que refiere el antecedente de una cirugía de columna lumbosacra por espondilolistesis L5-S1, 8 meses atrás. Luego del tercer mes comienza con mayor lumbalgia y pérdida de fuerza muscular en territorio L5. En radiografía y resonancia se constata migración posterior del dispositivo intersomático que ocupa el receso lateral y comienzo de foramen (Figura 3). 


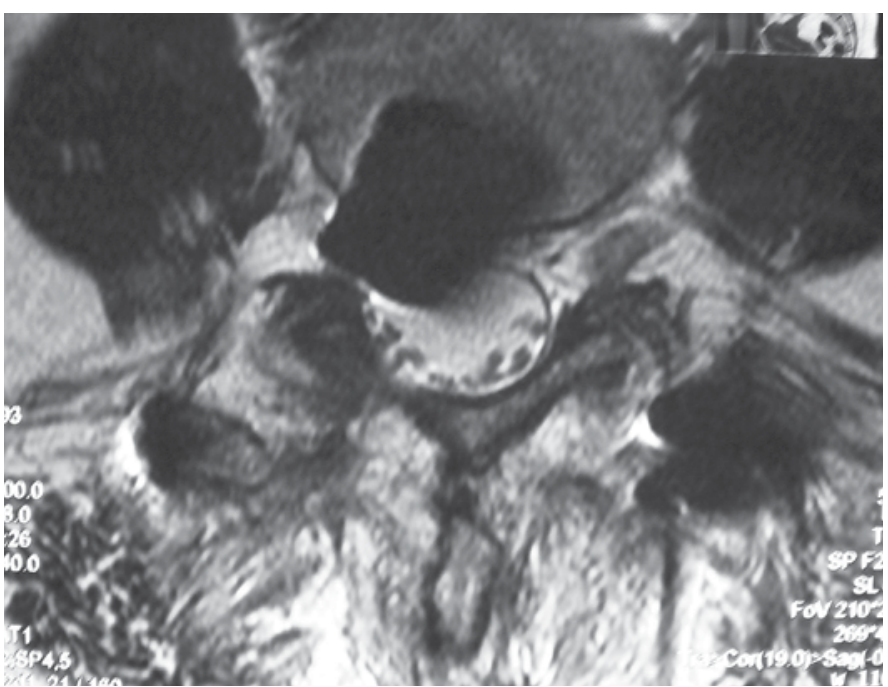

Figura 3. Corte Axial T2 que muestra la migración posterior del PLIF en el Pte. №3.

Nuestro manejo de esta complicación se realizó mediante el abordaje por vía anterior retroperitoneal, para realizar discectomía, retiro de material migrado y colocación de un dispositivo intersomático (ALIF) y asociación de ser necesario de abordaje posterior para el retiro del material de osteosíntesis, como se realizó en el primer caso que presentaba aflojamiento de los tornillos pediculares (Figura 4).
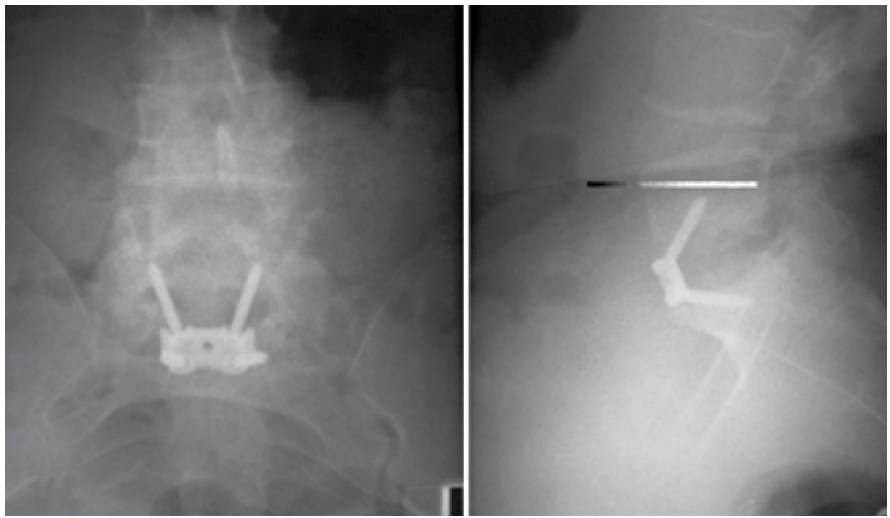

Figura 4. Imagen post-operatoria de la solución quirúrgica.

\section{RESULTADOS}

En nuestros casos el abordaje inicial cuidando el peritoneo y las estructuras retroperitoneales las realizó un cirujano general. Una vez protegidas las estructuras vasculares la realización de la discectomía y el retiro del material se ven facilitados por la ausencia de fibrosis cicatrizal.

Se realizó una búsqueda bibliográfica para analizar los datos sobre el retiro según parámetros de evidencia científica que se detallan en la Tabla 1.

En 1996, Glassman et al. ${ }^{8}$ reportan 1 paciente de 46 años que luego de un PLIF con caja trapezoidal y tornillos que presentó canal lumbar estrecho por migración. Para el retiro utilizaron la vía anterior (retroperitoneal), basándose en que al realizar una vía posterior en un paciente con cirugía previa podría, traumatizar más la raíz nerviosa y aumentar las disestesias.

Kuslich et al. ${ }^{9}$ en 1998 comparo 591 ALIF con 356 PLIF. Las migraciones que requirieron re operación fueron en total de 1,2\%.
La remoción o recolocación de los implantes se realizo dentro de los primeros 3 meses y la decisión dependió del cirujano.

Durante 1999 McAfee et al. ${ }^{10}$ describieron el manejo de revisión en 20 pacientes, en promedio a las 31 semanas, de artrodesis circunferencial por nueva sintomatología neurológica. Para los 4 casos con migración posterior aconsejan el retiro por vía posterior.

Tabla 1. Análisis según evidencia científica.

\begin{tabular}{|c|c|c|c|c|c|}
\hline $\begin{array}{l}\text { Autor y } \\
\text { año }\end{array}$ & $\begin{array}{l}\mathrm{N}^{\circ} \text { Casos } \\
\text { (Migrados) }\end{array}$ & Tto no Qco & $\begin{array}{l}\text { Retiro por } \\
\text { VA (justif) }\end{array}$ & $\begin{array}{c}\text { Retiro por VP } \\
\text { (Justif) }\end{array}$ & Evidencia \\
\hline $\begin{array}{c}\text { Glassman } \\
\text { (1996) }\end{array}$ & $\begin{array}{l}1 \text { (1) PLIF } \\
+ \text { TP }\end{array}$ & & $\begin{array}{c}1 \text { (Dificultad } \\
\text { por la fibrosis, } \\
\text { lesión dural } \\
\text { y tracción de } \\
\text { raíz). } \\
\text { ALIF }\end{array}$ & & IV \\
\hline $\begin{array}{l}\text { Kuslich } \\
\text { (1998) }\end{array}$ & $\begin{array}{c}591 \text { ALIF } \\
\text { (14) Vs } 356 \\
\text { PLIF (11) }\end{array}$ & $\begin{array}{c}\text { No } \\
\text { describre } \\
\text { (5) }\end{array}$ & \multicolumn{2}{|c|}{$\begin{array}{l}6 \text { PACIENTES dependiendo la } \\
\text { decisión del cirujano. }\end{array}$} & II \\
\hline $\begin{array}{c}\text { McAfee } \\
\text { (1999) }\end{array}$ & $\begin{array}{l}8 \text { ALIF + } \\
12 \text { PLIF } \\
\text { (4 PLIF) }\end{array}$ & & & $\begin{array}{c}4 \\
\text { [sin mover el } \\
\text { saco y la raíz] }\end{array}$ & III \\
\hline $\begin{array}{c}\text { Uzi } \\
(2001)\end{array}$ & $\begin{array}{l}2 \text { PLIF solo } \\
\text { (2) con } \\
\text { revisión } \\
\text { temprana }\end{array}$ & & & 2 & IV \\
\hline $\begin{array}{c}\mathrm{Ha} \\
(2004)\end{array}$ & $\begin{array}{c}10 \\
\text { infectados } \\
\text { TP + PLIF }\end{array}$ & $\begin{array}{l}\text { ATB + } \\
\text { Corsé }\end{array}$ & $\begin{array}{c}10 \text { (Dificultad } \\
\text { por la } \\
\text { fibrosis). } \\
\text { ALIF }\end{array}$ & Retiro TP & III \\
\hline $\begin{array}{l}\text { Chen } \\
\text { (2005) }\end{array}$ & $\begin{array}{c}118-88(3) \\
\text { Plif }+-\mathrm{TP}\end{array}$ & Corsé & & $\begin{array}{l}\text { ¿2? (solo habla } \\
\text { de retiro y } \\
\text { descompresión } \\
\text { a los } 3 \mathrm{~m} \text { ) }\end{array}$ & III \\
\hline $\begin{array}{l}\text { Nguyen } \\
\text { (2006) }\end{array}$ & $\begin{array}{l}14 \text { Ptes de } \\
\text { revisón - } 4 \\
\text { PLIF, } 5 \text { TLIF } \\
\text { y } 5 \text { ALIF } \\
\text { (3 PLIF y } 4 \\
\text { ALIF) }\end{array}$ & & $\begin{array}{c}14 \text { (por } \\
\text { lesiones } \\
\text { neurológicas } \\
\text { por VP, } \\
\text { cuidado con } \\
\text { lesiones } \\
\text { vasculares) }\end{array}$ & & II \\
\hline $\begin{array}{l}\text { Mirovsky } \\
\text { (2007) }\end{array}$ & $\begin{array}{c}8 \text { infecta- } \\
\text { dos (1) } \\
111 \text { PLIF }\end{array}$ & ATB & & $\begin{array}{l}1 \text { para recolo- } \\
\text { cación }\end{array}$ & III \\
\hline
\end{tabular}

Elias et al. ${ }^{11}$, en el 2000, analizaron retrospectivamente 67 pacientes instrumentados en 74 niveles solamente con PLIF y con un seguimiento de 12 meses. Describieron las complicaciones intraoperatorias con un $15 \%$ de lesiones de la duramadre y un 25\% que requirieron transfusión sanguínea. Las complicaciones postoperatorias fueron: 28 casos de dolor persistente, 10 casos de pseudoartrosis, 2 casos de migración posterior. 14 pacientes se re operaron colocando tornillos pediculares por continuar con dolor y/o inestabilidad demostrada radiológicamente y 2 pacientes con radiculopatía persistente y migración posterior, fueron removidos.

Un año después Uzi et al. ${ }^{3}$, reporta 2 casos de migración posterior temprana que fueron retirados por vía posterior.

En el año 2003, Scadutto et al. ${ }^{2}$ comparan en forma retrospectiva 31 PLIF consecutivos con 88 ALIF consecutivos, no asociados con fijación posterior, ingresados entre Enero 1996 a Junio 1998, todos con dispositivos cilíndricos buscando diferentes complicacio- 
nes. La muestra no es uniforme tanto en el número de pacientes como en el número de cirujanos que hacían los distintos abordajes (3 posteriores Vs 8 anteriores). Con respecto a las complicaciones concluye que: el rango global es de $22 \%$. PLIF $>$ ALIF $(p=0.001)$ en las intraoperatorias. PLIF > ALIF en POP mayores y ALIF > PLIF en menores, pero ambas sin significación estadística. Con respecto al tipo de complicaciones estas son abordaje dependiente, sin lesiones viscerales intraoperatorias en el ALIF. Las diferencias intraoperatorias: Sangrado PLIF $>$ ALIF $(p=0.001)$. Tiempo quirúrgico PLIF $>$ ALIF $(p=0.001)$. Tiempo de internación similar.

Durante el 2004 se observa el trabajo de Ha y $\mathrm{Kim}^{6}$ donde presenta la complicación infecciosa de la artrodesis circunferencial, en sus conclusiones aconsejan para evitar las lesiones neurológicas por la fibrosis cicatrizal el retiro de las cajas por vía anterior y posteriormente realizar un abordaje posterior para el retiro de los tornillos pediculares.

Chen et al. ${ }^{1}$ en el 2005 realizan un estudio retrospectivo para evaluar la migración de los PLIF en un total global de 118 pacientes solo completaron la evaluación 88, 51 hombres y 31 mujeres con espondilolistesis ístmicas, desde Octubre 1997 hasta Agosto 2000, utilizando PLIF con y sin instrumentación. En este trabajo las complicaciones fueron divididas en: 1- retropulsión (>2mm) y 2- subsidencia. Los 88 pacientes eran divididos si tenían o no instrumentación. La migración posterior se produjo en todos aquellos casos sin instrumentación (16,7\%) ( $\mathrm{p}$ 0.01). 3 pacientes presentaron retropulsión y 4 subsidencia a los 3 meses de post-operatorio. De los 3 con retropulsión 2 se localizaban en L4-L5 y el otro en L5-S1 y solo 2 debieron ser operados por severa sintomatología y por haber

fracasado el tratamiento ortopédico (Corsé). De los 4 con subsidencia a 2 se asocio fijación pedicular y otros 2 con tratamiento conservador.

En un estudio realizado por Nguyen et al. ${ }^{12}$ en el 2006, analiza retrospectivamente 14 pacientes (7 mujeres y 7 varones) interve- nidos quirúrgicamente entre 1998 y 2005 que requirieron revisión lumbar por vía anterior y evaluaron incidencia de complicaciones de esta vía. El tiempo promedio de revisión fue de 17 meses, en 7 oportunidades por migración de 3 PLIF y 4 ALIF. Concluyen diciendo que en las revisiones tanto de dispositivos colocados por vía anterior o posterior presentan altas tasas de complicaciones. El retiro por vía posterior presenta mayor tasa de lesiones neurológicas. La mayor pérdida sanguínea y mayor estadía hospitalaria se incrementan con las revisiones anteriores y la complicación vascular es 2 veces más alta en niveles L4-L5 que L5-S1. A pesar ello aconsejan el retiro por vía anterior.

Durante el año pasado, Mirovsky et al. ${ }^{13}$ realizaron un estudio retrospectivo de 20 pacientes con infección profunda en cirugías con PLIF con artrodesis posterolateral en 2 centros entre 1996 y 1999, con un seguimiento de 2 años luego del tratamiento de ATB. Un investigador independiente evaluó la recurrencia de infección, la artrodesis y satisfacción. De 111 pacientes con PLIF se infectaron 8 (6 con hueso y 2 con cajas). 6 pacientes requirieron Toilette, en un caso que la caja migró fue recolocada y se asoció con instrumentación posterolateral.

\section{CONCLUSIONES}

No existen publicaciones de Evidencia tipo 1 o 2 que marquen directivas claras

La presencia de fibrosis post-quirúrgica orientaría al abordaje por vía anterior en el retiro de los dispositivos intersomáticos y así evitar las lesiones del saco dural y la elongación radicular.

La dificultad del manejo vascular y el riesgo de eyaculación retrógrada en pacientes del sexo masculino son parámetros a tener en cuenta para optar por el abordaje posterior.

El retiro de los PLIF migrados a posterior, que causen sintomatología dolorosa y/o deficitaria se convierte una práctica altamente demandante y de manejo dificultoso.

\section{REFERÊNCIAS}

1. Chen $\mathrm{L}$, Yang $\mathrm{H}$, Tang T. Cage migration in spondylolisthesis treated with posterior lumbar interbody fusion using BAK cages. Spine (Phila Pa 1976). 2005;30(19):2171-5.

2. Scaduto AA, Gamradt SC, Yu WD, Huang J, Delamarter RB, Wang JC. Perioperative complications of threaded cylindrical lumbar interbody fusion devices: anterior versus posterior approach. J Spinal Disord Tech. 2003;16(6):502-7.

3. Uzi EA, Dabby D, Tolessa E, Finkelstein JA. Early retropulsion of titanium-threaded cages after posterior lumbar interbody fusion: a report of two cases. Spine (Phila Pa 1976). 2001;26(9):1073-5

4. Greiner-Perth $\mathrm{R}$, Boehm $\mathrm{H}$, Allam $\mathrm{Y}$, Elsaghir $\mathrm{H}$, Franke J. Reoperation rate after instrumented posterior lumbar interbody fusion: a report on 1680 cases. Spine (Phila Pa 1976). 2004:29(22):2516-20

5. Inamdar DN, Alagappan M, Shyam L, Devadoss S, Devadoss A. Posterior lumbar interbody fusion versus intertransverse fusion in the treatment of lumbar spondylolisthesis. J Orthop Surg (Hong Kong). 2006;14(1):21-6.

6. Ha KY, Kim YH. Postoperative spondylitis after posterior lumbar interbody fusion using cages. Eur Spine J. 2004;13(5):419-24.

7. Zhao J, Hou T, Wang X, Ma S. Posterior lumbar interbody fusion using one diagonal fusion cage with transpedicular screw/rod fixation. Eur Spine J. 2003;12(2):173-7.
8. Glassman SD, Johnson JR, Raque G, Puno RM, Dimar JR. Management of iatrogenic spinal stenosis complicating placement of a fusion cage. A case report. Spine (Phila Pa 1976). 1996;21(20):2383-6.

9. Kuslich SD, Ulstrom CL, Griffith SL, Ahern JW, Dowdle JD. The Bagby and Kuslich method of lumbar interbody fusion. History, techniques, and 2-year follow-up results of a United States prospective, multicenter trial. Spine (Phila Pa 1976). 1998;23(11):1267-78;

10. McAfee PC, Cunningham BW, Lee GA, Orbegoso CM, Haggerty CJ, Fedder IL, et al. Revision strategies for salvaging or improving failed cylindrical cages. Spine (Phila Pa 1976). 1999;24(20):2147-53.

11. Elias WJ, Simmons NE, Kaptain GJ, Chadduck JB, Whitehill R. Complications of posterior lumbar interbody fusion when using a titanium threaded cage device. J Neurosurg. 2000;93(1 Suppl):45-52

12. Nguyen HV, Akbarnia BA, van Dam BE, Raiszadeh K, Bagheri R, Canale S, et al. Anterior exposure of the spine for removal of lumbar interbody devices and implants. Spine (Phila Pa 1976). 2006;31(21):2449-53.

13. Mirovsky $Y$, Floman $Y$, Smorgick $Y$, Ashkenazi $E$, Anekstein $Y$, Millgram MA, et al. Giladi M. Management of deep wound infection after posterior lumbar interbody fusion with cages. J Spinal Disord Tech. 2007;20(2):127-31 